\title{
Studies on optical transmittance of boron-doped nanocrystalline diamond films
}

\author{
A. Wieloszyńska*1, R. Bogdanowicz ${ }^{1}$ \\ ${ }^{1}$ Faculty of Electronics, Telecommunications and Informatics, Gdańsk University of Technology, G. Narutowicza \\ 11/12, 80-233 Gdańsk
}

Received September 07, 2018; accepted September 21, 2018; published September 30, 2018

\begin{abstract}
Thickness is one of the most important parameters in many applications using thin layers. This article describes thickness determination of a boron-doped nanocrystalline diamond (NCD) grown on fused silica glass. A spectroscopic measurement system has been used. A high refractive index (2.3 at 550nm) was achieved for NCD films. The thickness of NCD samples has been determined from the transmission spectrum.
\end{abstract}

The number of applications of thin layer structures has increased because optoelectronics and microelectronics are developing. The structures can be used as protective and insulating elements. The thin layer structures are widely used also in optics, for example in construction of interference filters, sensors or dielectric mirrors, or as an antireflection layer [1-3]. In all the listed applications, it is important to control the thickness of the thin layer. The dielectric insulating layers require a minimum coating thickness to increase the resistance to the electric puncture. The thickness of optic structures, used as filters or an antireflective layer, must be specified to several nanometres. Solving the problem of thickness determination, including the selection of an appropriate measurement method, is not easy. The thickness measurement can be carried out ex-situ or in-situ [4]. In the latter, the influence of the technological process and the measuring apparatus should be taken into consideration when selecting an appropriate measurement method. The chosen method should be resistant to noise emitted during the synthesis of the layer and it should not affect the correct deposition of the coating.

An important group of methods for determining the thickness of thin layers are optoelectronic techniques, e.g. spectrophotometry, ellipsometry [4-5]. The measurements are based on registration and analysis of radiation reflected or transmitted by the layer. Some of these methods allow not only to determine the thickness $d$, but also the index of refraction $n$ and the coefficient of extinction $k$. This is particularly important when examining the layers used in optics.

Optical methods, compared to others, have many advantages, including high accuracy, high speed of

\footnotetext{
*E-mail: aleksandra.wieloszynska@pg.edu.pl
}

measurements, possibility of testing transparent and opaque layers, and direct, non-invasive and nondestructive nature of measurement. Optical spectroscopy is widely used to measure the thickness of thin transparent structures. The method determines thickness by using the reflection or transmission spectrum.

To show the method, we assume that radiation reflected from the dielectric layer deposited on a glass substrate is measured. Two beams - reflected from the layer and from the substrate - interfere with each other. If the phase between these beams is equal to $\beta=\pi / 2$, the first order minimum appears on the reflectance spectrum. The first order maximum appears if the phase difference is equal to $\beta=\pi$. The minima and maxima will appear for the phase differences equal to $\beta=\pi / 2,3 \pi / 2,5 \pi / 2 \ldots$ and $\beta=\pi, 2 \pi$, $3 \pi \ldots$, respectively. The example of reflectance with one minimum and maximum is shown in Fig. 1.

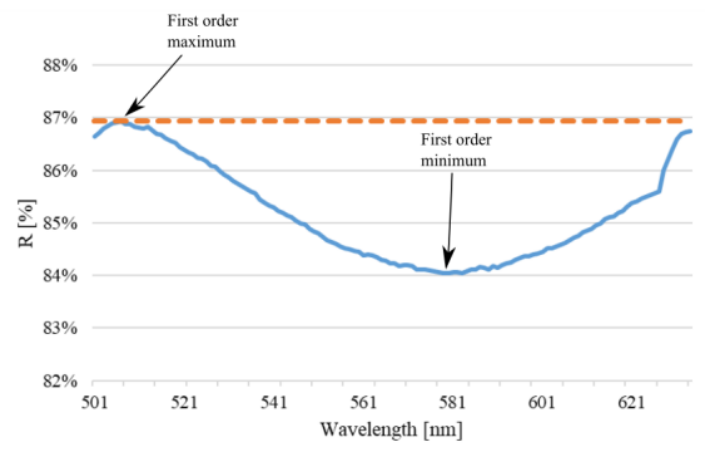

Fig. 1. Example of a thin layer reflectance $R$ spectrum.

If the thickness of the layer increases in time, the reflection considered for a particular wavelength would reach subsequent minima and maxima. Assuming energy conservation and low absorption in materials, reflectance $R$ can be represented as [6-7]:

$$
R=r_{1}+\frac{r_{1}+r_{2} \theta^{-2 i \delta}}{1+r_{1} r_{2} \theta^{-2 i \delta}} .
$$

The $r_{1}$ and $r_{2}$ are the Fresnel coefficients, and $\delta$ expresses the change in the phase of the beam passing through the layer and is described by the dependence: 


$$
\delta=\frac{2 \pi}{\lambda} n d \cos (\theta)
$$

Maxima and minima of the reflectance spectrum can be determined for the following values of optical thicknesses $n d$ :

$$
n d=(2 m+1) \frac{\lambda}{4} \text { and } n d=(2 m+2) \frac{\lambda}{4} .
$$

Assuming that the refractive index is constant and we know the values of the next maxima, optical thickness can be expressed by the dependence:

$$
n d=\frac{\lambda_{x} \lambda_{x+1}}{\lambda_{x}-\lambda_{x+1}},
$$

where: $d$ - the physical thickness of the layer, $\lambda_{x}$ and $\lambda_{x+1}$ - the wavelengths corresponding to the next maxima (or minima). To increase the accuracy of calculated values, dispersion should be taken into account.

If the difference between the refractive indices of the substrate and the layer increase, the difference between the maximum and the minimum increases too. In the case that the refractive index of the substrate and refractive index of the layer are, the similar determination of thickness is difficult.

Optical spectroscopy, due to the type of radiation, can be divided into ultraviolet, visible, near infrared, and infrared spectroscopy. The wavelength of radiation determines the range of the measured thickness. The shorter the waves of light are, the thinner the layers can be examined.

Spectroscopic methods enable non-contact and direct measurement. The practical accuracy of thickness measurements depends on the resolution of the measurement system. The main advantage of this type of method is that the measurements can be performed both in transmission and reflection mode. This allows you to use them for measurements in most technological chambers.

During the experiment, the thickness of boron-doped nanocrystalline diamond films on fused silica (NCD) has been determined. The diamond films were synthesized using MW PE CVD system (SEKI Technotron AX6200S, Japan). The samples were placed in a CVD chamber on a molybdenum stage.

The base pressure (before growth) in the vacuum chamber was $10^{-4}$ Torr. The working pressure was kept at 50 Torr. Methane $\left(\mathrm{CH}_{4}\right)$, diborane $\left(\mathrm{B}_{2} \mathrm{H}_{6}\right)$ and hydrogen $\left(\mathrm{H}_{2}\right)$ were used as the precursor gases. All the deposition parameters for each an NCD sample are listed in Table 1. During the process the molybdenum stage was heated up only to $500^{\circ} \mathrm{C}$ by an induction heater and controlled by a thermocouple. Plasma was generated with microwave radiation $(2.45 \mathrm{GHz}) 1300 \mathrm{~W}$, which is efficient for diamond synthesis. [8-9] After the growth process the substrate temperature was slowly reduced $\left(2^{\circ} \mathrm{Cmin}^{-1}\right)$ down to room temperature.

Table 1. Description of samples and the corresponding deposition parameters.

\begin{tabular}{|c|c|c|c|c|c|}
\hline sample & $\begin{array}{c}\mathrm{H}_{2} \\
{[\mathrm{sccm}]}\end{array}$ & $\begin{array}{c}\mathrm{CH}_{4} \\
{[\mathrm{sccm}]}\end{array}$ & $\begin{array}{c}\mathrm{B}_{2} \mathrm{H}_{6} \\
{[\mathrm{sccm}]}\end{array}$ & setup & $\begin{array}{c}\text { Time } \\
{[\mathrm{min}]}\end{array}$ \\
\hline NCD-1 & 292.5 & 3 & 7.5 & $\begin{array}{c}\text { cone-shaped } \\
{[10]}\end{array}$ & 180 \\
\hline NCD-2 & 292.5 & 3 & 7.5 & standard [11] & 60 \\
\hline
\end{tabular}

A spectroscopic measurement system was used to measure the thickness of thin transparent layers. It consists of a source, transmission track, and a spectrometer used to receive and analyze the spectrum. Figure 2 shows a diagram of the used system in the transmission mode.

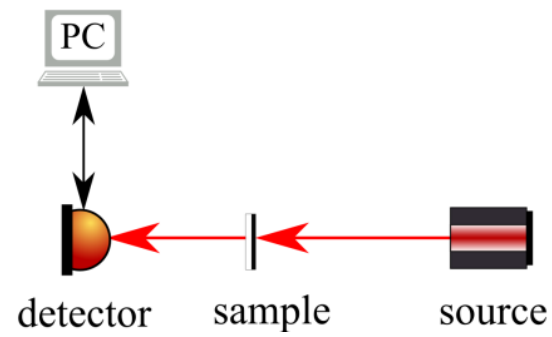

Fig. 2. Diagram of the measurement system.

The range of the analyzed radiation is strictly dependent on the used source. For example, a halogen lamp is used in measurements in the visible and near infrared range and can determine the thickness of layers thinner than $1 \mu \mathrm{m}$.

The specification of the used spectrometer influences measurement accuracy. The most important parameters are sensitive to spectrum characteristic, resolving power and characteristic of the dispersion.

First, reference measurement was made. It allowed us to determine the spectrum characteristic of the substrate and the track, and to compensate the non-linearity of the source. Then, the transmission spectrum of the tested sample was determined.

In Figure 3 the results of the measurement have been presented. The refractive index $n$ and determined thickness $d$ of the tested NCD samples are listed in Table 2. The measurement and simulated results are similar between $400 \mathrm{~nm}$ and $800 \mathrm{~nm}$. This is the most commonly used range of the wavelength in measurements. In ultraviolet there are some discrepancies which are the result of free carrier absorption. This absorption was not included in the simulation.

During the simulation it was assumed that we measure a monocrystalline diamond but in fact we measure polycrystalline crystals in which the inclusions of a non-diamond occur. The discrepancies occurred in the infrared are the result of those inclusions. 


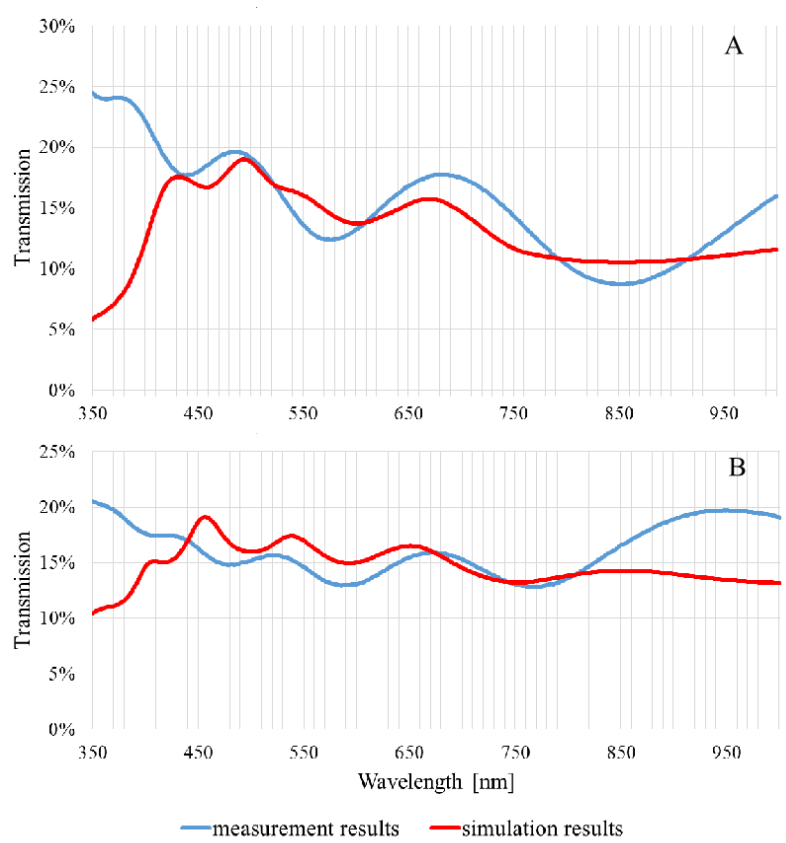

Fig. 3. Measurement and model transmission spectrum of the tested samples :A. NCD-1, B. NCD-2.

Table 2. Determined parameters of samples.

\begin{tabular}{|c|c|c|c|c|}
\hline sample & $\begin{array}{c}\text { refractive } \\
\text { index }(n)\end{array}$ & $\begin{array}{c}\text { Calculated } \\
\text { thickness } \\
(d)[\mathrm{nm}]\end{array}$ & $\begin{array}{c}\text { Reference } \\
\text { thickness } \\
(d)[\mathrm{nm}]\end{array}$ & $\begin{array}{c}\text { Growth } \\
\text { rate } \\
{[\mathrm{nm} / \mathrm{min}]}\end{array}$ \\
\hline NCD-1 & 2.3 & 286 & $246[10]$ & 1.59 \\
\hline NCD-2 & 2.3 & 557 & $530[5]$ & 9.28 \\
\hline
\end{tabular}

The achieved value of the refractive index is around 2.3. Similar values have been presented by Z.G Hu et al. [5, 12]

The growth rates for both samples differ significantly from each other. A special cone-shaped shim used in the deposition process has influenced the growth rate on the NCD-2, slowing the kinetics of the CVD process as reported previously in Ref. [10].

In conclusions, thickness determination of an NCD film has been presented in this letter. This parameter is very important for many applications. To achieve the thickness of NCD films, a spectroscopy measurement system has been used. The simulated and measured transmission of the samples were compared. The results are similar in the visible range.

During the growth of diamond films a special truncated cone-shaped shim and a normal shim were used for both samples, respectively. The obtained parameters are compared with the values from literature.

The authors gratefully acknowledge the financial support from the Polish National Science Centre (NCN) under
Grant No. 2016/21/B/ST7/01430, 2016/22/E/ST7/00102 and National Centre for Science and Development Grant Techmatstrateg No. 347324. The DS funds of the Faculty of Electronics, Telecommunications, and Informatics of the Gdansk University of Technology are also acknowledged.

\section{References}

[1] Z. Li, S. Butun, K. Aydin, ACS Photonics 2(2), 183 (2015).

[2] L. Yu, D.D. Tune, C.J. Shearer, J.G. Shapter, Solar Energy 118, 592 (2015).

[3] D. Majchrowicz et al., Materials 11(1), 109 (2018).

[4] K.L. Konnerth, F.H. Dill, IEEE Trans. Electron. Dev. 22(2), 452 (1975).

[5] Z.G. Hu, P. Prunici, P. Hess, K.H. Chen, J. Mater. Scien.: Mater. Electron. 18(1), 37 (2007).

[6] J. Adamczewska et al., Procesy technologiczne $w$ elektronice pótprzewodnikowej (WNT, Warsaw 1980).

[7] J.P. Dilger, L.R. Fisher, D.A. Haydon, Chem. Phys. Lipids 30(23), 159 (1982).

[8] M. Ficek et al., Appl. Surf. Scien. 387, 846 (2016).

[9] R. Bogdanowicz et al., J. Opt. Soc. Korea 19, 705 (2015).

[10] M. Sobaszek et al., Opt. Mater. 42, 24 (2015).

[11] R. Bogdanowicz et al., Diamond Related Mater. 55, 52 (2015).

[12] Z.G. Hu, P. Hess, Appl. Phys. Lett. 89(8), 081906 (2006). 